\title{
PréSence éditoriale du Nouveau roman en Pologne
}

\author{
Elżbieta SKIBIŃSKA \\ Université de Wrocław (Pologne)
}

\begin{abstract}
En): The aim of this paper is to study the factors of the intraduction (Ganne, Minon 1992) of works written by the nouveau roman authors to the Polish literary space. The translation is understood here as the publication, i.e. the result of the publishing process which determines the diffusion of the text in the target market (Wilfert-Portal, Guérin 2012). After a bibliometric (quantitative) survey of the Polish translations of nouveau roman novels, we submitted its results to qualitative interpretations (also taking into account the editorial peritexts) which showed that there is a relatively regular, though not systematic, transfer of nouveau roman works to Poland. Two periods may be observed. The first begins in 1958, the year of the first translation, and ends in 1989, the year of the political transformations in Poland. During this period, translations were published by prestigious state houses, whose production depended on political and ideological factors. The second period, beginning in 1990, was marked by the end of the highly politicised organization of culture in Poland and by the transition to free-market economy methods. Novels by nouveau roman writers are now dispersed between various publishers and translators, and their intraduction, which follows mechanisms dictated by the progressive integration of the country into global networks, seems to be motivated by the publishers' search for symbolic and economic capital.
\end{abstract}

Keywords (En): nouveau roman; Polish translation; publishing market

Mots-clés (Fr): nouveau roman; traduction polonaise; marché éditorial

\section{Introduction}

Par la formulation du titre de cette étude, je tiens à souligner que ma réflexion porte sur les œuvres des auteurs du mouvement Nouveau Roman qui sont traduites en langue polonaise et publiées en Pologne.

La traduction est donc comprise ici comme publication, ou résultat de l'activité éditoriale qui conditionne le fonctionnement public - la diffusion - du texte ${ }^{1}$. Il s'agit ainsi de l'activité d'un ensemble d'acteurs - auteurs, traducteurs, éditeurs régie par des facteurs autres que purement littéraires; en effet, la production éditoriale dépend des éléments de nature économique, culturelle et politique qui déterminent les flux de traduction : leur volume, leur direction et leur « contenu ». Cette façon de considérer la traduction en fait un « objet sociologique », dans le sens défini par SAPIRO et HEILBRONN (2002), et aussi - c'en est une facette - un moyen de transfert interculturel, soumis principalement à l'action des facteurs propres au contexte de réception des œuvres traduites.

\footnotetext{
${ }^{1} \mathrm{Cf}$. à ce propos : «Les traductions dont parlent les études de traduction sont en effet, dans la très grande majorité des cas, d'abord des livres, des articles, des chapitres, des extraits de livres et plus généralement des pages imprimées. Avant que d'être des systèmes de signes justiciables d'une lecture esthétique, idéologique ou linguistique qui décide d'en ignorer la forme matérielle, elles sont des objets la plupart du temps marchands. » (WILFERT-PORTAL, GUÉRIN 2012 : 51).
} 
Le but que je me pose est d'analyser l'intraduction ${ }^{2}$ des nouveaux romanciers dans l'espace littéraire polonais et de chercher à dégager certains facteurs qui régissent l'inscription dans cet espace de ce groupe d'auteurs qui, pendant un temps, ont marqué la vie littéraire française par une recherche esthétique particulière.

Pour ce faire, je commencerai par présenter les résultats d'un examen bibliométrique $^{3}$ des notices réunies dans une liste bibliographique des traductions polonaises des œuvres des nouveaux romanciers publiées sous forme de livre (sont donc exclues des analyses les traductions d'extraits publiées dans des revues ${ }^{4}$ ). Des constatations de nature quantitative sur leur présence éditoriale en Pologne servent de point de départ à des observations qualitatives concernant les principaux acteurs qui participent à l'intraduction des nouveaux romanciers en Pologne : les éditeurs. Je m'intéresse particulièrement aux choix éditoriaux concernant la façon dont l'œuvre est proposée au public: son inclusion dans une collection et l'éventuelle présence de péritextes éditoriaux (quatrième de couverture, préfaces, postfaces...) dans la mesure où leur contenu peut indiquer l'appartenance de l'oeuvre ou de l'auteur au mouvement ${ }^{5}$. Il faut préciser en même temps que - puisque je m'intéresse ici à l'intraduction de l'oeuvre dans le nouvel espace littéraire, mais non à sa place et son fonctionnement dans cet espace - je ne m'occupe pas des épitextes (critiques, débats dans la presse, interviews ou entretiens avec les auteurs) ${ }^{6}$.

La première question qu'il est nécessaire de se poser lors de la constitution d'une liste bibliographique qui sert de base à des analyses est celle des critères qui permettent d'y faire entrer tel auteur ou telle œuvre. Selon Anthony PYM, Sandra POUPAUD et Ester TORRES SIMÓN (2009), le premier critère est l'objet à prendre en compte comme « traduction». Comme je l'ai dit plus haut, celle-ci est comprise ici comme ouvrage publié en tant que traduction, c'est-à-dire contenant dans sa description bibliographique la mention 'traduit de' et, le plus souvent, le nom du traducteur.

Le critère suivant est l'appartenance de l'auteur de l'oeuvre traduite au courant du Nouveau Roman. C'est le premier filtre qui m'a permis de sélectionner les œuvres à inclure dans ma liste. Et c'est là que le premier problème est apparu.

\footnotetext{
${ }^{2}$ L'« intraduction» signifie l'importation littéraire sous forme de traduction, 1 '« extraduction », l'exportation sous forme de traduction ; termes empruntés à GANNE et MiNON (1992: 58).

${ }^{3}$ Le terme de «bibliométrie » (bibliometrics) a été proposé en 1969 par Alan Pritchard qui l'a défini comme «application des méthodes mathématiques et statistiques aux livres et autres moyens de communication » (PritchaRd 1969 : 4 ; cité d'après SKALSKA-ZLAT 1993 : 6). Depuis, le terme a été souvent redéfini, mais la définition de Pritchard en reste la base, les divers ajustements servant surtout à souligner que la bibliométrie, qui se pratique à partir des bibliographies, permet de saisir et caractériser divers mécanismes régissant le processus de la communication écrite.

${ }^{4}$ Telle la traduction de passages de Beckett dans la revue Literatura na Świecie (mai 1975, avril 1981), de Simon dans la même revue (novembre-décembre1986) ou Duras dans Kwartalnik Artystyczny: Kujawy i Pomorze (février 2011).

${ }^{5}$ Pour accéder au contenu des péritextes éditoriaux, il a été nécessaire de soumettre à l'examen également les livres. L'analyse approfondie des péritextes est prévue pour une autre étude.

${ }^{6}$ Ils ont fait l'objet d'une étude de Michał Piotr Mrozowicki (2003).
} 
En effet, si l'appellation de Nouveau Roman ${ }^{7}$, consacrée en quelque sorte par le titre du numéro spécial de la revue Esprit de juillet-août 1958, existe bien et ne semble pas poser problème, "établir la liste exacte des nouveaux romanciers est déjà en soi une gageure », comme le constate Nelly Wolf dans le premier chapitre de son livre Une littérature sans histoire : essai sur le nouveau roman (WOLF 1995 : 32). Intitulé «1950-1960, l'impossible définition », ce chapitre rend bien compte des difficultés à déterminer « la véritable identité collective du Nouveau roman » (WOLF 1995 : 40) et à recenser les auteurs qui font partie du groupe des nouveaux romanciers ${ }^{8}$.

Dans ce travail, j'ai pris comme base le numéro cité de la revue Esprit (1958), la célèbre photo devant le siège des Éditions de Minuit $(1959)^{9}$, et la liste des romanciers qui ont participé au colloque de Cerisy-la-Salle, organisé par Jean Ricardou (1971) : ce sont les « repères » le plus souvent utilisés pour inventorier les membres de ce «collectif littéraire dont l'existence n'a jamais été prouvée » (Wolf 1995 : 9).

En puisant dans ces «documents »- témoignages de l'adhérence, même temporaire, à cette « école du refus » (PINGUAUT, 1958) - j' ai choisi de former une variante large, ou inclusive, de la liste des nouveaux romanciers : Samuel Beckett, Michel Butor, Jean Cayrol, Marguerite Duras, Jean Lagrolet, Claude Mauriac, Claude Ollier, Robert Pinget, Alain Robbe-Grillet, Nathalie Sarraute, Claude Simon, Kateb Yacine, soit les auteurs qui apparaissent au moins dans une des trois sources indiquées ci-dessus. Il s'agit donc d'écrivains dont le nom, à un moment ou un autre, a été associé à l'émergence du courant et à son histoire ${ }^{10}$, même si certains (tel Butor ou Pinget) ont, comme on le sait, cessé d'écrire de la fiction, et d'autres (Duras, Simon), sortis du Nouveau Roman, ont bâti une œuvre indépendante qui leur a valu une consécration individualisée (WOLF $1995: 9)^{11}$.

\footnotetext{
${ }^{7}$ L'expression, initialement péjorative, a été utilisée par le critique Émile Henriot dans un compterendu de La jalousie et de la réédition des Tropismes publié dans Le Monde en mai 1957.

${ }^{8}$ Voir aussi : DUgast-Portes (2001 : 3-59); DENÈs (2001); DidiER (2001).

${ }^{9}$ Voir FAERBER (2004) ; sur le rôle de l'éditeur dans la formation du Nouveau Roman, voir SiMONIN (1996).

${ }^{10}$ Francine Dugast-Portes (2001 : 229-232) donne dans son livre une chronologie des oeuvres des nouveaux romanciers qui compte les auteurs suivants (le nombre entre parenthèses indique celui des titres publiés, y compris les écrits à caractère théorique) : Baudry (5), Beckett (13), Butor (21), Duras (34), Faye (5), Mauriac (11), Ollier (17), Pinget (18), Ricardou (7), Robbe-Grillet (16), Roche (6), Sarraute (13), Simon (23), Sollers (19). Il est frappant d'observer que les noms des auteurs postérieurs à la période de la formation du mouvement (ici en italiques) sont tous absents des bibliographies et catalogues analysés pour les besoins de mon travail : si leurs œuvres ont été traduites, c'est seulement sous forme d'extraits publiés dans des revues (telle Literatura na Świecie).

${ }^{11}$ Sur les rapports entre Duras et le courant de Nouveau Roman voir Jiřr Šrámek (1975). Voir aussi la déclaration de F. Dugast-Portes : „Soulignons au passage que nous choisissons de traiter l'oeuvre de Duras dans le présent volume : quelques années plus tard, elle ne vient pas au premier grand colloque de Cerisy, ou se retrouvaient ceux que Jean Ricardou allait désormais définir comme les «nouveaux romanciers », c'est-à-dire, outre lui même, Michel Butor, Claude Ollier, Robert Pinget, Alain RobbeGrillet, Nathalie Sarraute, Claude Simon; mais Robbe-Grillet intervint souvent pour affirmer la parenté littéraire de «Marguerite » avec le groupe. Quant à Beckett, qui appartient à une autre génération, son nom apparaît sans cesse dans les interventions" (DUGAST-PORTES, $2001: 5-6)$.
} 
Cette liste inclusive pourrait être opposée à celle, plus restreinte, que l'on peut établir à partir des considérations de Michał Piotr Mrozowicki : en effet, dans son étude de la réception du Nouveau Roman par la critique polonaise, il parle de Butor, Robbe-Grillet, Sarraute, Simon, Claude Mauriac, Ollier et Pinget ; il mentionne aussi Duras comme «proche du Nouveau Roman » (MrozowICKI 2003 : 345) ; il ne mentionne pas Cayrol et Beckett, qui sont cependant considérés comme précurseurs du mouvement ${ }^{12}$ et cités comme tels dans certaines sources polonaises, par exemple dans l'article « Nouveau roman » du Stownik literatury polskiej XX wieku (1992: 712).

Qui cherche à établir la liste des traductions des œuvres des nouveaux romanciers se heurte cependant à une autre difficulté : l'instabilité ou le caractère évolutif de la poétique de ces auteurs. En effet, si certaines de leurs œuvres peuvent être classées comme nouveaux romans, d'autres n'entrent pas dans cette catégorie :

Entre un début généralement encore assez traditionaliste et le tournant hypermoderniste négocié à la fin des années cinquante, la plupart des nouveaux romanciers sont passés par un style intermédiaire caractérisé par la suppression des marques de l'intentionnalité, l'altération de la chaîne des causalités, et le retrait de l'intrigue derrière l'énigme (WOLF 1995 : 34).

C'est d'autant plus vrai que, indépendamment de l'année prise comme référence de l'«officialisation » du courant - 1953 (parution des Gommes de Robbe-Grillet) ou 1958 (publication du numéro d'Esprit) - chacun des futurs nouveaux romanciers avait déjà à l'époque une ou plusieurs œuvres publiées à son actif ${ }^{13}$. D'autre part, certains - on l'a déjà dit - ont abandonné ensuite les principes de l'esthétique du $\mathrm{NR}^{14}$.

C'est donc une liste inclusive des auteurs et de toutes leurs œuvres qui a constitué le point de départ de mon inventaire des traductions publiées en polonais sous forme de livre. Il embrasse la production éditoriale de la période allant de 1958

${ }^{12}$ Laissons la parole à la spécialiste, auteur d'un manuel universitaire : «Soulignons au passage que nous choisissons de traiter l'œuvre de Duras dans le présent volume : quelques années plus tard, elle ne vient pas au premier grand colloque de Cerisy, ou se retrouvaient ceux que Jean Ricardou allait désormais définir comme les «nouveaux romanciers ", c'est-à-dire, outre lui même, Michel Butor, Claude Ollier, Robert Pinget, Alain Robbe-Grillet, Nathalie Sarraute, Claude Simon; mais RobbeGrillet intervint souvent pour affirmer la parenté littéraire de « Marguerite » avec le groupe. Quant à Beckett, qui appartient à une autre génération, son nom apparait sans cesse dans les interventions » (Dugast-Portes 2001 : 5-6). Sur le rôle de Beckett : «Molloy et Malone sont tout à fait des Nouveaux Romans, et qui mériteraient la carrière de Godot» (Robbe-Grillet, Le Voyageur, cité dans le Dictionnaire Beckett, HUBERT, 2011 : 503).

${ }^{13}$ Il suffit de citer comme exemple Marguerite Duras, auteure de La vie tranquille (1944) ou d'Un barrage contre le Pacifique (1950), de facture traditionnelle, publiés avant Moderato Cantabile (1958, aux Éditions de Minuit, considéré comme une des œuvres les plus réussies du Nouveau Roman). Sur les trajectoires créatives et esthétiques des nouveaux romanciers voir Wolf (1995 : 15-16 et 54-63).

${ }^{14}$ On peut donner comme exemple Claude Mauriac - auteur de La Terrasse de Malagar, mais aussi Marguerite Duras, auteure de La maladie de la mort ou de La douleur, 1985, qui échappent à l'esthétique du NR. Un problème plus compliqué encore est le caractère bilingue des œuvres de Beckett : certaines ont été écrites en anglais et autotraduites en français (tels Soubresauts), d'autres (tel Premier amour), écrites en français. Pour mes analyses, j'ai choisi les œuvres dont la traduction polonaise s'est faite à partir du français (conformément à l'information fournie dans les catalogues et dans le livre même). 
(première traduction publiée) jusque 2016 (publication de la dernière traduction). Il contient moins de noms d'auteurs (Jean Lagrolet, Claude Ollier et Kateb Yacine en sont absents), et naturellement, moins de titres qu'une éventuelle liste de toutes les œuvres considérées comme «nouveaux romans » : si la Chronologie proposée par Francine Dugast-Portes contient deux cent huit titres (cent soixante-treize, si l'on ne compte pas ceux des auteurs venus dans les années 60), ma liste en comprend trentecinq (y compris quatre romans de Jean Cayrol, absent de la Chronologie).

Il est d'autant plus intéressant de voir quels auteurs et titres ont été sélectionnés pour être introduits dans l'espace littéraire polonais, et de quelle manière ils ont été présentés par l'éditeur responsable de leur intraduction. Le recours à la liste large (inclusive) des auteurs ouvre la possibilité de confronter les avis des critiques et théoriciens avec la pratique éditoriale. En effet, situer une œuvre dans une collection, la doter d'un péritexte éditorial, c'est aussi avertir le lecteur de l'appartenance de l'auteur à un mouvement, et celle de l'œuvre à un genre.

La liste des traductions polonaises des œuvres des nouveaux romanciers a été dressée à partir des données contenues dans les bibliographies des traductions ${ }^{15}$ et dans les catalogues en ligne de trois bibliothèques: la Bibliothèque Nationale polonaise, la Bibliothèque de l'Université de Wroclaw et la Bibliothèque Jagellonne ${ }^{16}$.

La liste contient les données suivantes : titre français de l'ouvrage original, titre de la traduction, année de publication de l'original, année de publication de la traduction, nom du traducteur, éditeur de la traduction, lieu de publication de la traduction, nom de la collection, présence d'une préface ou postface.

\section{1. Étude quantitative}

\subsection{La place des nouveaux romanciers dans l'espace littéraire polonais}

La présence polonaise des nouveaux romanciers est à considérer dans un cadre plus large, celui de la place de la littérature française intraduite dans l'espace polonais, ce qui demande une mise en contexte de la littérature étrangère intraduite en Pologne.

Le graphique 1 montre que jusqu'en 1990, les traductions du français publiées en Pologne se situent après celles de l'anglais et du russe ${ }^{17}$; après 1990 , c'est l'anglais qui est la langue-source dominante (entre 70 et $80 \%$ des traductions publiées), alors que le français et l'allemand deviennent «concurrents » à la $2^{\mathrm{e}}$ ou $3^{\text {e }}$ place. Ce qui devrait être remarqué, c'est le volume de traductions du français

\footnotetext{
${ }^{15}$ Bibliografia literatury ttumaczonej na język polski wydanej w latach 1945-1976, I, 1977, Czytelnik, Warszawa; Bibliografia literatury ttumaczonej na język polski wydanej w latach 1977-1980, III, 1983, Czytelnik, Warszawa.

${ }^{16} \mathrm{Ce}$ sont toutes des bibliothèques auxquelles, conformément à la loi, tous les éditeurs polonais doivent envoyer un «exemplaire obligatoire» de chaque titre publié. Malgré cette loi, certains éditeurs ne s'exécutent pas, et les données collectées doivent donc être traitées avec une certaine réserve, surtout celles qui concernent la période d'après 1989 .

${ }^{17}$ Ces données se rapportent aussi bien aux nouvelles traductions qu'aux rééditions de traductions existantes (d'auteurs anciens ou contemporains).
} 
dans la période 1956-1960 : c'est là qu'il est le plus important par rapport aux autres langues sur toute la période étudiée.

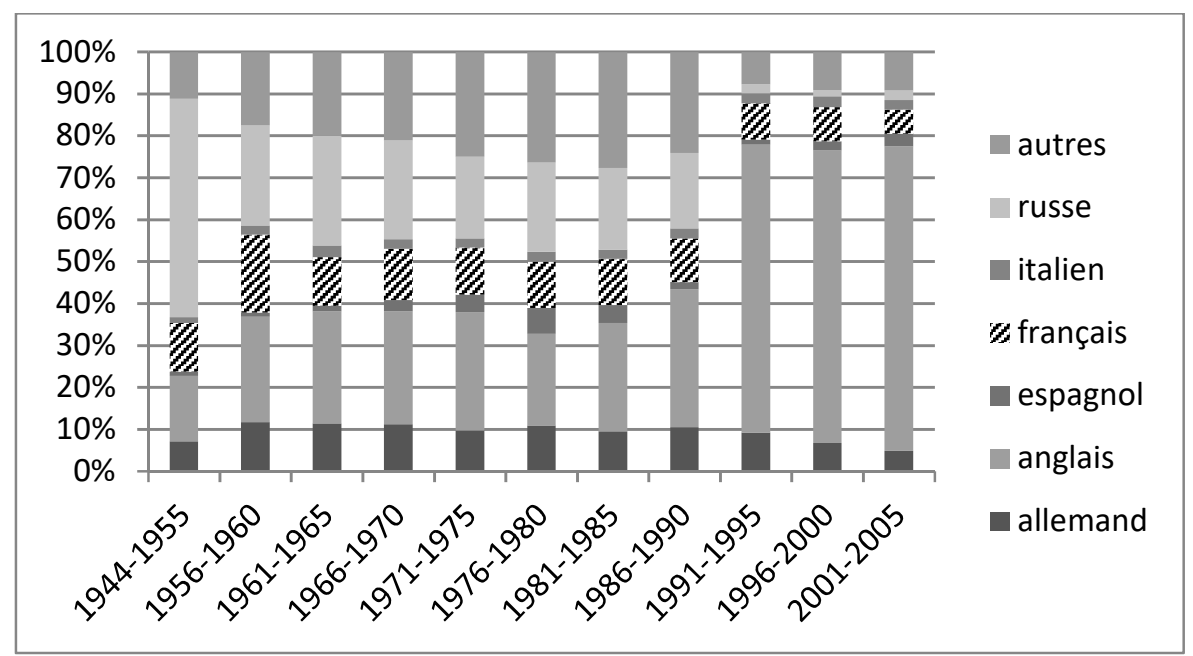

Graphique 1 : Volumes de traductions de belles-lettres (1944-2005) (par langues), en pourcentage ${ }^{18}$

Pour notre analyse, ceci est d'autant plus important que c'est précisément dans cette période que sont publiées en polonais les premières œuvres classées dans le mouvement du Nouveau Roman, comme le montre le graphique 2 :

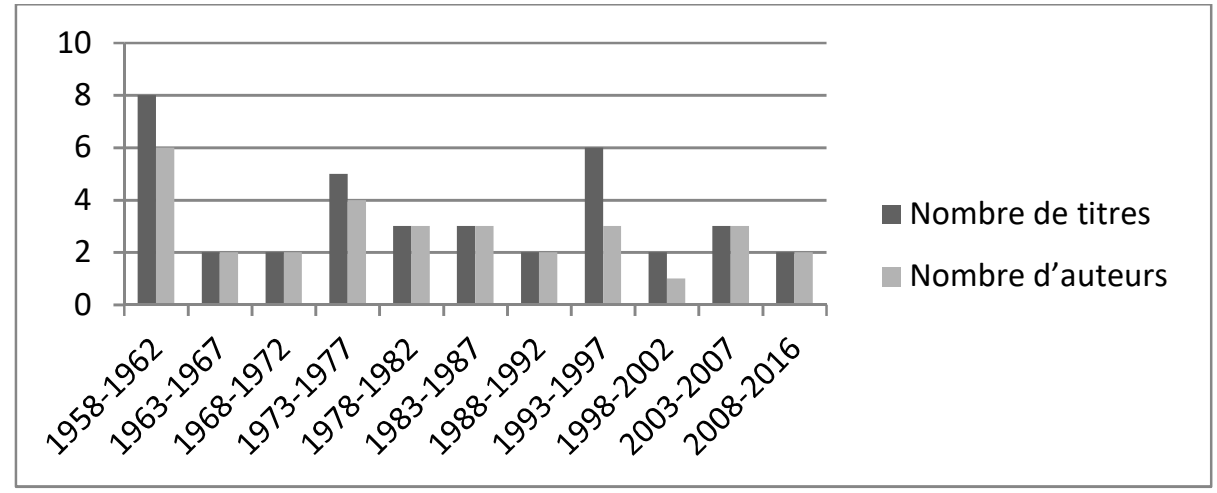

Graphique 2 : Volumes de traductions d'œuvres appartenant au Nouveau Roman (auteur; titre), en saisie chronologique

\footnotetext{
${ }^{18}$ Graphique basé sur les données qui proviennent de l'annuaire Ruch Wydawniczy w liczbach (Polish Publishing in Figures) publié par la Bibliothèque Nationale polonaise depuis l'année 1955. Attention à la barre des années 1944-55, qui couvre dix ans alors que toutes les autres en couvrent cinq. 
On remarque que si la moyenne par période de cinq ans est de deux ou trois titres, trois «quinquennats» se distinguent par des pics marquants : 58-62 (témoignant le plus probablement de l'intérêt porté au nouveau phénomène de la littérature française, mais aussi d'une certaine libéralisation de la production éditoriale, résultat de la situation politique du pays - voir plus loin), 73-77 (les années qui suivent les réformes économiques et politiques introduites dès 1971 par Edward Gierek, premier secrétaire du Parti ouvrier unifié polonais) et 93-97 (les années qui suivent la chute du régime communiste et qui marquent le passage à l'économie libérale).

Mais - le graphique 2 en rend compte aussi - les nouveaux romanciers sont toujours présents dans la production éditoriale, même si le nombre de titres est très bas. Ce qui nous amène à nous demander quels auteurs et quelles œuvres continuent d'être traduits alors que le mouvement lui-même s'est éteint.

\subsection{Les auteurs traduits}

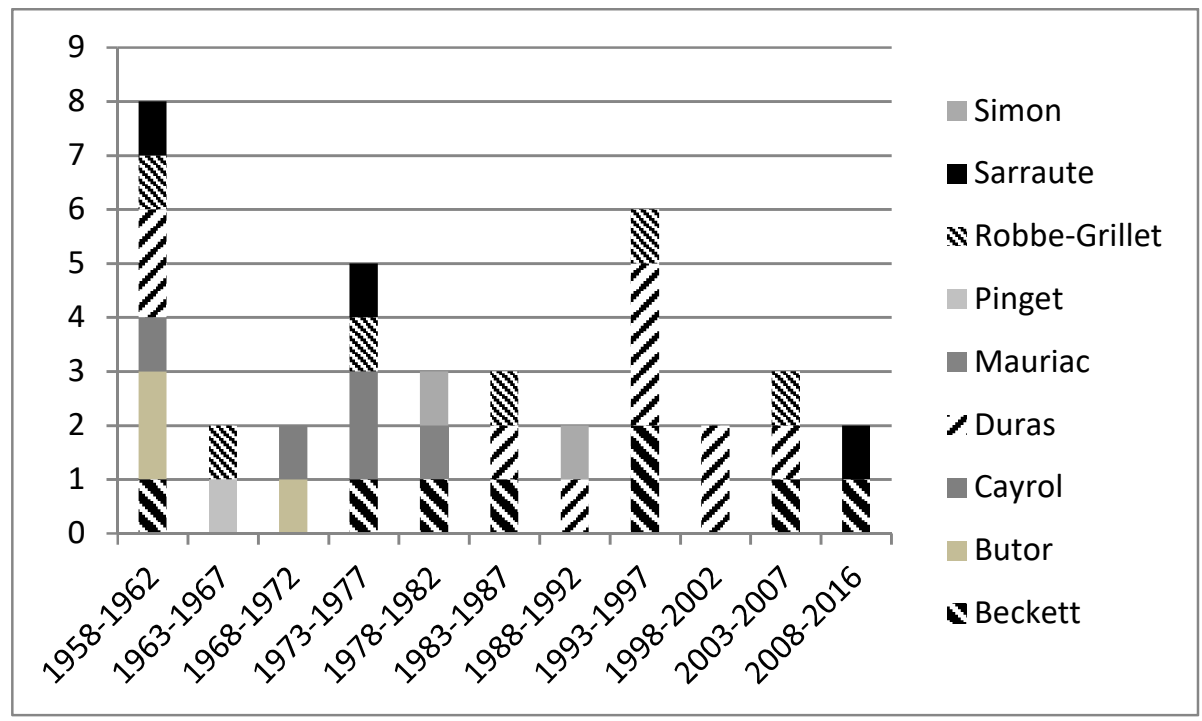

Graphique 3a : Nombre de titres publiés par auteur (saisie chronologique) 


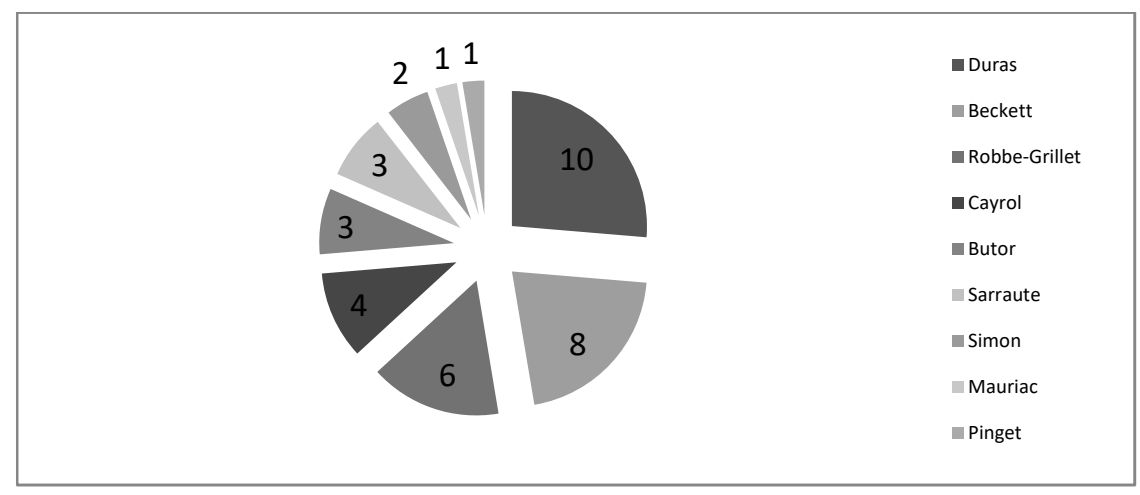

Graphique $3 b$ : Nombre de titres publiés par auteur

Les graphiques $3 \mathrm{a}$ et $3 \mathrm{~b}$ montrent que ce sont les œuvres de Marguerite Duras (10) et de Samuel Beckett (8) qui sont le plus publiées, mais aussi, qui intéressent toujours les éditeurs, puisqu'ils continuent à les proposer au public polonais. Ce sont aussi leurs livres qui ont fait l'objet de retraductions (L'Amant; Molloy) ou de réédition(s) (L'Amant dans la traduction de Loda Kałuska a été publié 3 fois par trois éditeurs différents : WL 1989 ${ }^{19}$, WAB 1993, Znak 2007 ; Premier amour : WL 1973, 1994 ; Molloy WL 1983, 1994 ; on peut remarquer qu'en 1994, les deux récits paraissent chez cet éditeur dans un même volume, dans la collection ABC Klasyka dla każdego 'ABC Classiques pour tous').

Après ces deux auteurs - rappelons-le : considérés par certains comme proches du Nouveau Roman, mais n'appartenant pas au «noyau dur » du courant - vient son chef de file et auteur emblématique, Alain Robbe-Grillet : chaque décennie depuis 1958 voit la traduction (ou la réédition : Les gommes, Czytelnik 1959, Wyd. Tako 2007) d'un de ses romans. On peut remarquer aussi la traduction très récente (2016) d'un livre de Nathalie Sarraute, chose d'autant plus frappante qu'il s'agit des Tropismes, considérés comme l'ouvrage fondateur du Nouveau Roman. Ajoutons tout de suite qu'il a été publié - soixante-sept ans après la publication de l'original! - dans la collection Klasyka z Europy ('Classiques européens'), subventionnée par l'Union Européenne.

\subsection{Les éditeurs}

Quels sont les éditeurs polonais qui ont choisi de mettre dans leur catalogue des œuvres des nouveaux romanciers?

\footnotetext{
${ }^{19}$ La publication sous forme de livre a été précédée de la publication en feuilleton dans l'hebdomadaire cracovien Życie Literacki ('Vie littéraire').
} 


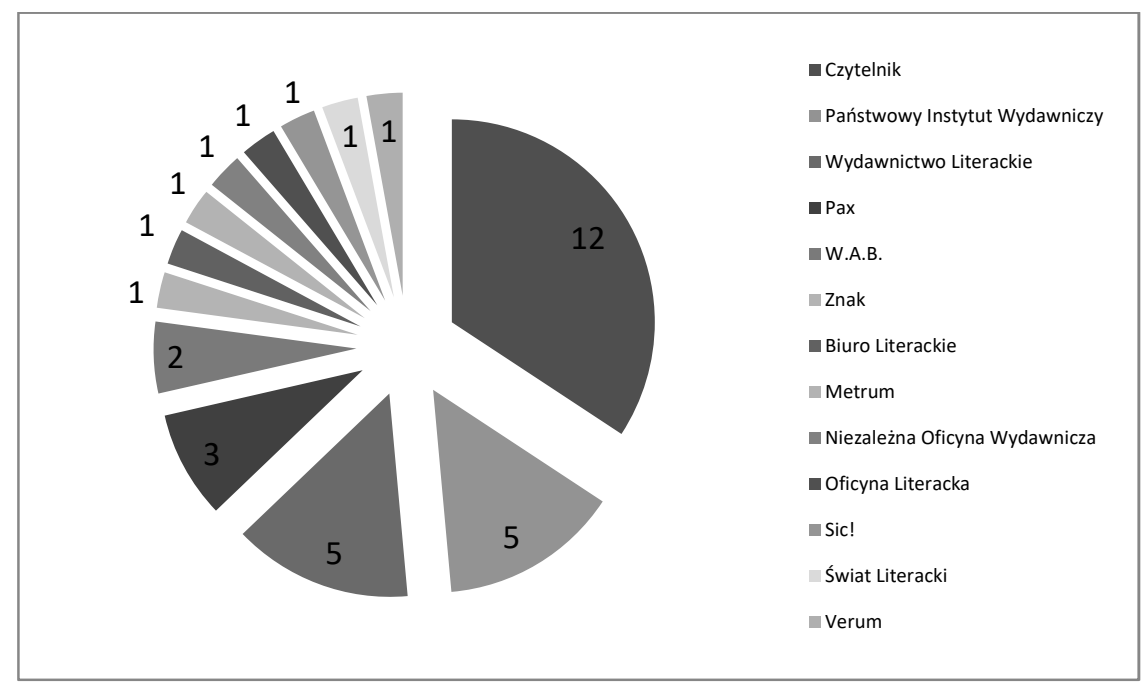

Graphique 4a : Nombre de titres publiés par éditeur

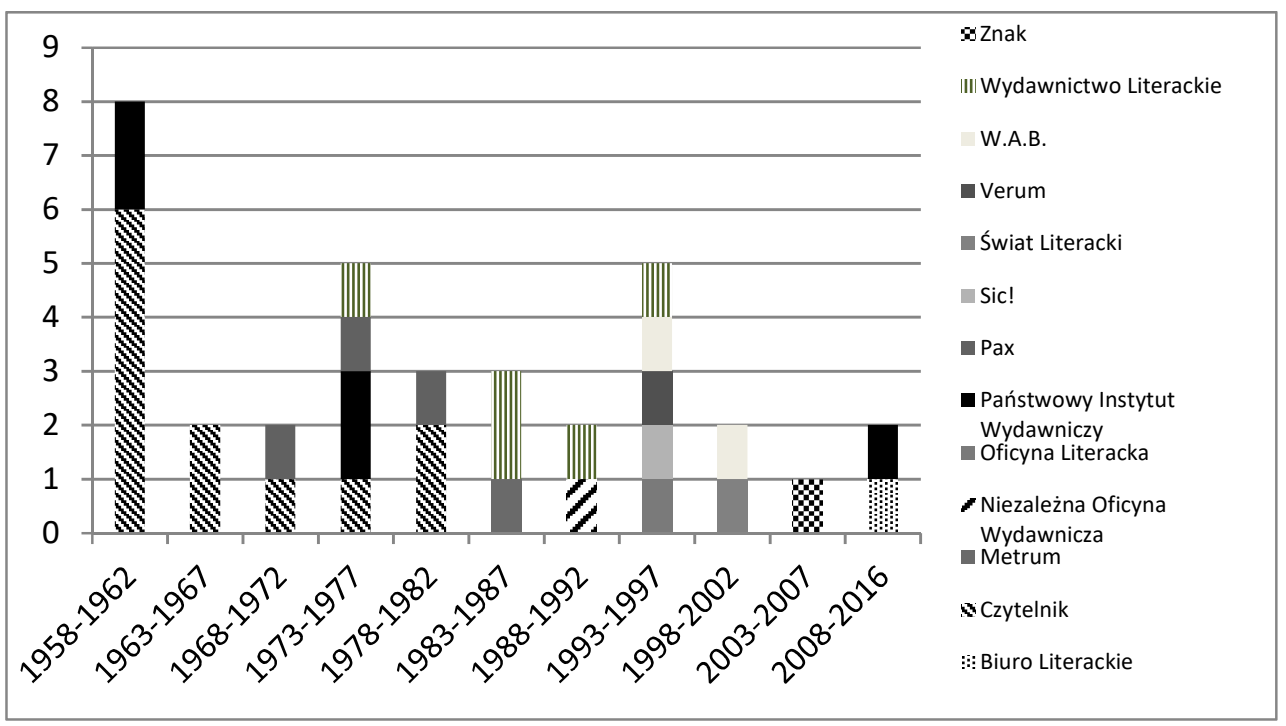

Graphique $4 \mathrm{~b}$ : Nombre de titres publiés par éditeur (saisie chronologique)

Les données que présente le graphique 4a montrent la part importante de titres publiés par la maison Czytelnik (douze, donc plus d'un quart de la totalité des titres publiés). On voit aussi que sept éditeurs - donc plus de la moitié - n'ont qu' un titre à leur actif. La saisie chronologique quant à elle (graphique 4b) confirme d'une part la concentration des titres chez Czytelnik (huit dans les années qui correspondent à la période d'activité la plus vive du mouvement) et révèle la dispersion des titres entre les éditeurs (treize titres sortis chez dix éditeurs) à partir des années 1988- 
1992, c'est-à-dire surtout après la libéralisation du marché éditorial en 1989. On constate enfin dans cette période la disparition de la plupart des maisons qui avaient jusque là publié les œuvres des nouveaux romanciers.

Ce phénomène est à situer dans le contexte plus général du fonctionnement du marché éditorial, et de l'intraduction vue comme un élément de ce marché.

\section{Observations qualitatives}

Revenons donc au volume de traductions de belles-lettres dans la saisie chronologique.

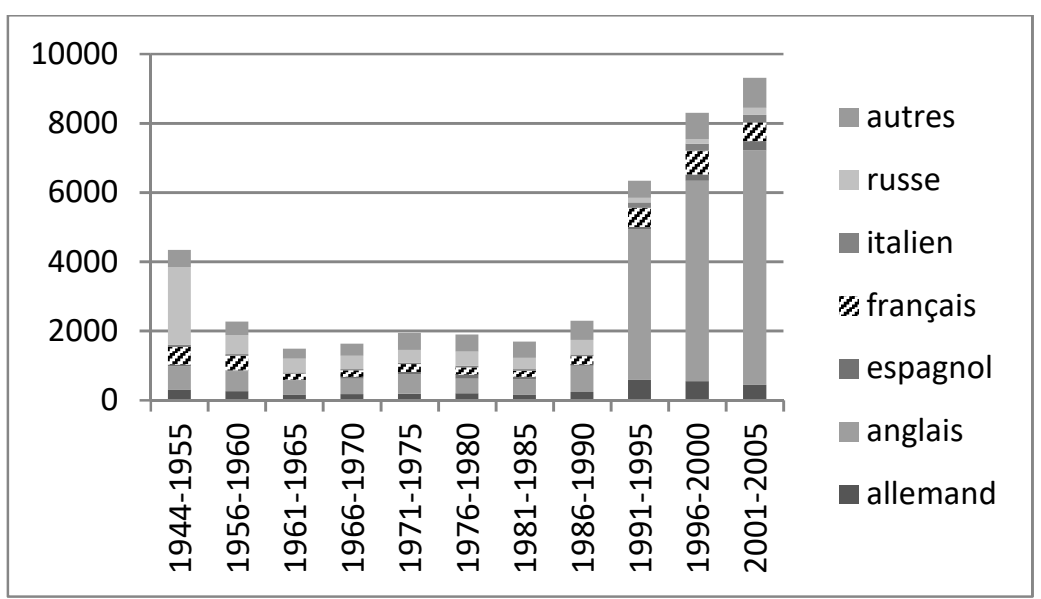

Graphique 5 : Volumes de traductions de belles-lettres (1944-2005) (par langues), en nombre absolu

Le graphique 5 montre un décalage frappant entre la moyenne annuelle de traductions avant et après 1990 (entre trois cents et quatre cents titres par an dans la période précédant cette date, et plus de mille deux cents dans les années qui suivent). Cet écart correspond naturellement à la chute du régime communiste en 1989, événement qui a profondément transformé tous les domaines de la vie des Polonais ; il a aussi modifié le marché du livre. Il convient de rappeler ici la différence fondamentale entre les périodes 1944-1989 et 1989-2016: dans la première, la soumission de l'activité des maisons d'édition, qui appartenaient à l'État, à des contraintes de nature idéologique et politique et aux règles de la planification centralisée (réalisation de plans correspondant au profil de l'éditeur); dans les années suivant 1989, l'obéissance aux règles du marché libre.

Pour mieux saisir les mécanismes qui régissent l'intraduction du Nouveau Roman, il semble nécessaire de rappeler aussi que les années 1956-1960 (et la décennie qui suit) - période de la plus forte intraduction du français - correspondent à une (ré)animation des relations franco-polonaises à partir de 1954, qui a conduit, entre autres, à la signature d'une convention de coopération culturelle et scientifique en 1957 (JAROSZ, PASZTOR, 2008 : 432 et suiv.). Elle a débouché, notamment, sur des séjours plus fréquents des Polonais en France et un accès plus facile aux revues littéraires françaises en Pologne, ce qui a facilité le contact direct avec la vie 
littéraire parisienne (dont les débats autour du nouveau phénomène qu'était le Nouveau Roman $)^{20}$.

Le passage de l'économie planifiée au mode de fonctionnement propre à l'économie de marché en 1989 transforme radicalement le monde de l'édition. Il signifie la disparition du carcan de la censure et de la planification centralisée, mais aussi la nécessité de se mouvoir sur un marché régi par la concurrence pour financer son activité, puisque l'État ne paie plus. C'est ainsi que l'on assiste à l'émergence immédiate et massive de petites maisons privées, parmi lesquelles on peut remarquer les presses parallèles (clandestines) créées avant 1989, dotées d'un fort capital symbolique, et parfois aussi d'un capital économique, qui rejoignent désormais l'espace légal. On observe aussi la présence grandissante de capitaux étrangers. Quant aux maisons d'État, un certain nombre d'entre elles disparaissent, souvent pour des raisons économiques, parce qu'elles ont été incapables de se restructurer.

La traduction, dans cette première période, est un moyen d'accumuler du capital symbolique, mais aussi économique - surtout pour les nouvelles maisons. Il faut aussi rappeler l'apparition de diverses formes de subventions, dont - pour les traductions des auteurs français -, le programme «Boy-Żeleński» dans les années $1990^{21}$.

Cette situation diamétralement opposée des éditeurs avant et après 1989 peut servir d'argument pour expliquer la présence des nouveaux romanciers telle qu'elle apparaît dans le tableau 1 (Annexe).

D'abord, chose non négligeable, on peut voir que dès la première décennie qui a suivi le dégel politique de 1956, le lecteur polonais a eu accès aux œuvres de Butor (L'emploi du temps, La modification), Beckett (Nouvelles et textes pour rien), Robbe-Grillet (Les gommes, La maison de rendez-vous), Cayrol (Le déménagement), Duras (Dix heures et demie du soir en été), Pinget (Autour de Mortin), Sarraute (Portrait d'un inconnu); il faut souligner que si le roman de Sarraute a attendu onze ans sa publication en polonais, et Les gommes six ans, pour les autres ouvres, l'intervalle entre la publication de l'original et celle de la

\footnotetext{
${ }^{20}$ Voir à ce propos Mrozowicki (2003: 339) : «En 1958, dans la revue Wspótczesnośćc, un jeune critique polonais, Konrad Eberhardt, relate son entretien avec Robbe-Grillet aux éditions de Minuit. Encore deux ans plus tôt, une telle rencontre, et à plus forte raison, une telle relation dans la presse polonaise, auraient été inimaginables. Mais après le tournant de 1956, le public polonais peut enfin découvrir de nouvelles tendances dans la littérature occidentale. Parmi ces découvertes, il y a, bien entendu, le Nouveau Roman que les critiques polonais de l'époque préfèrent appeler plutôt anti-roman ou roman réiste. Le dégel dans la vie politique - et culturelle - de la Pologne coïncide parfaitement avec la phase décisive de formation du Nouveau Roman et avec la publication de quelques-unes de ses œuvres-phares ou manifestes, qui ont provoqué, en France, et à l'étranger, un débat très animé. »

${ }^{21}$ Programme s'inscrivant dans l'initiative du ministère des Affaires étrangères français de créer, en 1990, des Programmes d'aide à la publication (PAP) pour «concilier les impératifs de la politique éditoriale de chaque éditeur et la promotion des auteurs français », comme on peut le lire dans la préface du rapport «Lire les auteurs français à l'étranger. Les programmes d'aide à la publication » (http://www.diplomatie.gouv.fr/fr/IMG/pdf/pap2005.pdf; consulté le 28 février 2018). Selon les données de ce rapport, environ 350 ouvrages traduits et publiés en polonais auraient bénéficié de cette aide ; parmi les auteurs qui nous intéressent ici, on trouve Duras (publiée par W.A.B., Świat Książki et Sic!) et Beckett (chez Wydawnictwo Literackie). Sur les fonctions de la traduction sur le marché éditorial polonais après 1989 voir SKIBIŃSKA (2009).
} 
traduction est en général de deux ans. Il n'est pas sans intérêt de rappeler les tirages de ces livres, qui dépassaient les dix mille exemplaires (et vingt mille dans le cas de La maison des rendez-vous, La jalousie ou La route de Flandres.... $)^{22}$.

La rapidité de la traduction témoigne de l'intérêt porté à l'actualité de la littérature française. La publication était accompagnée de critiques dans la presse, mais aussi - dans les revues spécialisées - d'analyses approfondies aussi bien des œuvres traduites que de celles qui ne l'étaient pas, mais qui suscitaient en France des débats sur le renouveau du roman ${ }^{23}$. Il ne faudrait pas non plus oublier le fait que les nouveaux romanciers étaient aussi des cinéastes (scénaristes), et que certains de leurs films étaient diffusés dans les salles polonaises, souvent presque en même temps qu'en France. Ainsi, Hiroshima mon amour (1960), Moderato Cantabile (1960), L'Année dernière à Marienbad (1961)... ont-il pu susciter l'intérêt du public pour les œuvres littéraires de leurs scénaristes.

J'ai déjà signalé la forte concentration des publications chez Czytelnik : douze titres (dont ceux de Butor, Robbe-Grillet, Duras, Pinget, Cayrol...) dans la période d'avant 1989. À cette époque, quatre autres éditeurs publient onze titres : PIW fait paraître deux romans de Butor, un de Cayrol et un de Sarraute ; Pax, deux romans de Cayrol et La Terrasse de Malagar de Mauriac ; et enfin WL, Premier amour et Molloy de Beckett, Djinn de Robbe-Grillet et L'Amant de Duras. Ce dernier est une retraduction, la première version polonaise étant sortie deux ans auparavant chez un petit éditeur clandestin et étant restée pratiquement inaccessible et inconnue ${ }^{24}$.

S'il est impossible de parler d'exclusivité d'un éditeur pour certains auteurs, une observation plus détaillée des pratiques éditoriales permet de constater l'existence d'éléments révélateurs d'une certaine action «programmatique », ou d'une intention de faire découvrir au lecteur le phénomène du Nouveau Roman.

Ceci concerne avant tout deux maisons: Czytelnik et Państwowy Instytut Wydawniczy (PIW). Czytelnik, fondée en 1944, avait pour vocation l'expansion et la propagation des connaissances et des œuvres littéraires; à partir de 1950, elle devient «spécialiste» des belles-lettres, en publiant en particulier la littérature contemporaine polonaise et étrangère. PIW a été fondé en 1946, avec le rôle de publier, en principe, de grandes œuvres des classiques polonais et étrangers ${ }^{25}$.

Il n'est donc pas étonnant que c'est chez ces éditeurs que sortent les livres des nouveaux romanciers. En effet, La maison de rendez-vous, La jalousie et La route de Flandres sont publiés dans la prestigieuse collection Nike, qui réunit sous un format de poche les traductions de prose du $\mathrm{XX}^{\mathrm{e}}$ siècle de Czytelnik; Les Corps étrangers et Vous les entendez entrent dans la collection (aussi prestigieuse) Współczesna Proza Światowa (Prose contemporaine mondiale) de PIW. Ils sont accompagnés - comme la plupart des romans publiés hors collection - de péritextes

\footnotetext{
${ }^{22}$ Les informations sur les tirages proviennent des livres mêmes. Avant 1989, les éditeurs étaient obligés de les indiquer dans tout ouvrage publié.

${ }^{23}$ L'étude citée de Mrozowicki en témoigne de façon magistrale. Voir aussi SIEKIERSKI (1992).

${ }^{24}$ Dans son étude sur la réception polonaise de Duras, Tomasz Stróżyński semble confondre les deux traductions et les traite comme une seule (STRÓŻYŃSKI 2005 : 94).

${ }^{25}$ Il faut souligner que les deux éditeurs ont joué, avant1989 (Czytelnik continue à le faire), un rôle important dans la vie littéraire polonaise, et leurs collections, ont contribué à la canonisation des œuvres et des auteurs.
} 
éditoriaux (couverture, postfaces ou préfaces de personnalités littéraires reconnues, comme Adam Ważyk) qui contiennent des informations - parfois très développées, parfois de simples mentions - sur l'appartenance de l'auteur à ce mouvement et sur les caractéristiques de son écriture ou de son esthétique. Il me semble intéressant de signaler que même La Terrasse de Malagar de Claude Mauriac, $3^{\mathrm{e}}$ partie de son journal consacrée à François Mauriac, est dotée d'une préface de la traductrice qui rappelle les liens de l'auteur avec le nouveau roman. Des remarques semblables accompagnent aussi Le déménagement de Cayrol et Molloy de Beckett (présenté comme auteur qui se situe au « seuil » du Nouveau Roman). On pourrait considérer que dans cette première période, un lien, ne serait-ce que faible, d'une oeuvre ou de son auteur avec le courant du Nouveau Roman, constitue un «label de qualité » littéraire.

Dans les années 1970 et 1980, la présence des nouveaux romanciers s'affaiblit ; si l'on continue de publier «l'actualité » (Djinn, L'Amant), on observe de plus en plus souvent le rattrapage de retards : on fait paraitre des œuvres dont l'original a été publié dix-huit (La jalousie), vingt-quatre, voire trente-deux ans auparavant. Cela est dû dans une certaine mesure à l'épuisement du mouvement lui-même et au rétrécissement du répertoire des œuvres à traduire. Mais il ne faut pas non plus oublier que, pour des raisons politiques et surtout économiques, les années 80 n'ont pas été une période propice à la traduction de nouveautés en Pologne.

J'ai déjà signalé la disparition, après la libéralisation du marché éditorial en 1989, de la plupart des maisons qui avaient précédemment publié les œuvres des nouveaux romanciers, et la dispersion des titres entre plusieurs éditeurs. On peut voir aussi que pendant cette période, ce sont les noms de Beckett et Duras qui dominent, avec Robbe-Grillet dans leur sillage: leurs œuvres sont rééditées, retraduites (Beckett), mais aussi, connaissent de premières traductions qui relèvent du rattrapage (Le voyeur, ou Moderato Cantabile, respectivement onze et quarante ans après la publication de l'original ; rappelons que le second est considéré comme un exemple de nouveau roman par excellence). Simon et Sarraute (Les tropismes) sont également traduits, et Robbe-Grillet est réédité (Les gommes).

Parmi les nouveaux éditeurs se trouvent des maisons qui investissent dans le capital symbolique, de relativement grandes (W.A.B., Znak) ou de petites maisons de niche qui publient des œuvres soigneusement sélectionnées (Oficyna Literacka, Sic!), mais aussi des maisons pratiquement inconnues qui surprennent par leur décision de mettre au catalogue un roman tel que Le voyeur (Verum) ou Les gommes (réédition en 2007 chez Wydawnictwo Tako, qui publie plutôt des livres pour enfants).

La présence des auteurs énumérés ci-dessus dans les catalogues de ces éditeurs peut être perçue comme une manifestation des mécanismes plus généraux observables après la chute du régime communiste : soumission à des critères de rentabilité, sans risque de pertes important, accumulation de capital économique (rééditions de L'Amant, connu grâce au film et pour ses aspects érotiques), accumulation de capital symbolique (traduction de «classiques» reconnus, lauréats des prix littéraires...). En témoignent aussi les péritextes éditoriaux, très différents de ceux de la période d'avant 1989 ; il se réduisent le plus souvent à la quatrième de couverture dont la visée est de convaincre et de provoquer 
la décision d'achat, et ceci, en un nombre de mots (ou d'informations) très limité. Ainsi, si l'on rappelle l'appartenance de l'œuvre au Nouveau Roman, comme dans le cas du Voyeur ou de Moderato Cantabile, c'est très discrètement, par allusion, en soulignant plutôt la position de l'auteur dans la littérature mondiale, et non pas française ; on évoque le fait que le roman a été porté à l'écran (Moderato Cantabile, L'Amant) ou encore on indique les prix qui lui ont été attribués (tel le Goncourt 1984 pour $\left.L^{\prime} A m a n t^{26}\right)$.

\section{Conclusion}

L'examen de la présence éditoriale des nouveaux romanciers dans l'espace littéraire polonais me conduit aux constatations suivantes :

(a) Trente-cinq titres traduits et publiés depuis 1958 (soit en soixante ans) ne constituent qu'une faible partie du volume inscrit dans la Chronologie proposée par Francine Dugast-Portes (même si l'on enlève de sa liste les auteurs venus après 1960). On ne peut que donner raison à Michał Piotr Mrozowicki :

Par ailleurs la liste des Nouveaux Romans qui, au moment où nous écrivons cet article, en mai 2002, ne sont pas (encore ?) traduits en polonais est presque interminable. Par conséquent, la majorité des lecteurs polonais, à l'exception d'un groupe plutôt restreint - hélas - de francophones, devait - et doit toujours - pour se faire une idée sur le Nouveau Roman, faire confiance aux jugements de critiques, ce qui est d'autant plus difficile que ces jugements, très souvent, sont contradictoires. (MROZOWICKI, $2003: 346$ ).

(b) Deux approches et deux étapes se laissent distinguer, et elles sont liées aux bouleversements politiques de 1989. Avant cette année, l'intraduction du Nouveau Roman se fait selon les règles de la production éditoriale soumise aux facteurs politiques et idéologiques. La coïncidence des changements entraînés par le dégel politique, de la reprise des relations culturelles entre la France et la Pologne au milieu des années 50 et de l'apparition d'un nouveau phénomène littéraire en France semble une raison suffisante pour expliquer le nombre relativement élevé des traductions, qui suivent pour la plupart l'actualité, surtout dans la première décennie. La baisse de volume observée à partir des années 70 peut avoir comme cause aussi bien l'essoufflement du mouvement Nouveau Roman que des raisons spécifiques à l'édition polonaise (économiques, mais aussi proprement littéraires qui devraient être analysées à part).

(c) L'activité éditoriale régie par les règles du marché libre impose un choix attentif des titres à publier; en ce qui concerne les nouveaux romanciers, leurs œuvres - qui appartiennent déjà à une époque révolue de la vie littéraire française sont rares et traitées comme des «classiques »; leur présence au catalogue peut donc contribuer à améliorer l'image de l'éditeur sans entraîner de risque sérieux de pertes économiques; les informations véhiculées par les péritextes éditoriaux, souvent très succinctes (principalement la $4^{e}$ de couverture), y contribuent en soulignant la qualité littéraire de l'ouvrage (lauréat d'un prix, souvent traduit, porté à l'écran).

\footnotetext{
${ }^{26} \mathrm{Il}$ est à remarquer que les éditions précédentes ne sont pas mentionnées dans le livre publié par Znak.
} 
(d) Les observations présentées ici ne concernent que la « surface visible », sous forme de chiffres ou des mentions contenues dans les péritextes éditoriaux. Une étude des matériaux cachés, conservés dans les archives des maisons d'édition et/ou des traducteurs serait nécessaire pour mieux comprendre les enjeux qui, à des époques différentes, motivaient les stratégies des entrepreneurs culturels ${ }^{27}$ que sont les éditeurs qui, par leurs décisions concernant la publication des traductions, introduisent et propagent de nouveaux éléments (étrangers) dans la culture d'accueil.

\section{BIBLIOGRAPHIE}

Bibliografia literatury ttumaczonej na język polski wydanej w latach 1945-1976, I (1977), Warszawa, Czytelnik.

Bibliografia literatury tłumaczonej na język polski wydanej w latach 1977-1980, III (1983), Warszawa, Czytelnik.

DENÈS Dominique (2001), Le nouveau roman: problématique d'une institutionnalisation, in: Le temps des lettres: Quelles périodisations pour l'histoire de la littérature française du 20e siècle? Rennes, Presses universitaires de Rennes. En ligne : <http://books.openedition.org/pur/33313>. ISBN : 9782753545731. DOI : 10.4000/books.pur.33313. (généré le 14 mai 2017).

DIDIER Alexandre (2001), Dater le nouveau roman? in : Le temps des lettres: Quelles périodisations pour l'histoire de la littérature française du 20e siècle? Rennes, Presses universitaires de Rennes. En ligne: $<$ http://books.openedition.org/pur/33314>. ISBN : 9782753545731. DOI : 10.4000/books.pur.33314. [généré le 14 mai 2017].

DugAst-PORTES Francine (2001), Le Nouveau Roman. Une césure dans l'histoire du récit, Paris, Nathan Université.

Even-ZOHAR, Itamar (2010), Papers in Culture Research, Tel Aviv, Unit of Culture Research, Tel Aviv University. En ligne: $<$ http://citeseerx.ist.psu.edu/viewdoc/download?doi=10.1.1.477.787\&rep=rep1 \&type $=$ pdf $>$ [consulté le 24 juillet 2018].

FAERBER Johan (2004), La photo Minuit, du cliché nocturne à la lumière du négatif, Fabula Les colloques. En ligne: http://www.fabula.org/colloques/document64.php [consulté le 28/02/2018].

GANNE Valérie, MinON Marc (1992), Géographie de la traduction, in : BARRETDUCROCQ Françoise (éd.), Traduire l'Europe, Paris, Payot, p. 55-95.

GŁOWIŃSKI Michał (1992), Nouveau roman, in : Stownik literatury polskiej XX wieku, Wrocław, Ossolineum.

HEILBRON Johan; SAPIRO Gisèle (2002), La traduction littéraire, un objet sociologique, Actes de la recherche en sciences sociales 144, (Traductions: les échanges littéraires internationaux), Paris, Seuil, p. 3-5

Hubert Marie-Claude (éd.) (2011), Dictionnaire Beckett, Paris, H. Champion.

${ }^{27}$ Terme emprunté à EvEN-ZoHAR (2010 : 194-198). 
JAROSZ Dariusz ; PASZTOR Maria (2008), Stosunki polsko-francuskie 1944-1980, Warszawa, PISM.

Lire les auteurs français à l'étranger. Les programmes d'aide à la publication (2005), en ligne: http://www.diplomatie.gouv.fr/fr/IMG/pdf/pap2005.pdf [consulté le 28 février 2018].

MrOZOWICKI Michał Piotr (2003), Les échos polonais du Nouveau Roman français, Revue de littérature comparée $\mathrm{n}^{\circ}$ 307, Paris, Klincksieck, p. 339-353.

PINGUAUT Bernard, L'école du refus, Esprit, juillet-août 1958.

PRITCHARD Alan (1969), Statistical bibliography or bibliometrics, Journal of Documentation, 4 (cité d'après : SKALSKA-ZLAT Marta (1993), Bibliometryczne badania rozwoju dyscypliny naukowej, Wrocław, Wydawnictwo Uniwersytetu Wrocławskiego.

PYM Anthony; POUPAUd Sandra; TORRES SIMÓN Ester (2009), Finding Translations. On the Use of Bibliographical Databases in Translation History, Meta 542, Montréal, Les Presses de l'Université de Montréal, p. 264-278.

SIEKIERSKI Stanisław (1992), Książk literacka : potrzeby społeczne i ich realizacja w latach 1944-1986, Warszawa, Wydawnictwo Naukowe PWN.

SiMONIN Anne (1996), La littérature saisie par l'histoire. Nouveau Roman et guerre d'Algérie aux Éditions de Minuit, Actes de la recherche en sciences sociales, Vol. 111-11 (Littérature et politique), Paris, Seuil, p. 59-75.

SKIBIŃSKA Elżbieta (2006), La traduction au service de l'idéologie: "Liste des lectures françaises" en polonais dans les années 1946-1960, in : ÜbersetzenTranslating-Tratuire: Towards a "Social Turn"?, Wolf Michaela (éd.), WienBerlin, LIT Verlag, p. 131-141.

SKIBIŃSKA Elżbieta (2009), La place des traductions sur le marché éditorial polonais après 1989, in : Les contradictions de la globalisation éditoriale, Sapiro Gisèle (éd.), Paris, Nouveau Monde éd., p. 335-367.

ŠRÁMEK Jiří (1975), Le Nouveau Roman et Marguerite Duras, Romanica Wratislaviensia XI, Wrocław, Wydawnictwo Uniwersytetu Wrocławskiego, p. 57-71.

STRÓŻYŃSKI Tomasz (2005), La réception de Marguerite Duras en Pologne, in : Marguerite Duras : l'existence passionnée. Actes du Colloque de Potsdam 1824 avril 2005, CIESLAK Benjamin ; BEIGEL Yves, Potsdam, Universitätsverlag, p. $90-96$.

WILFERT-PORTAL Blaise ; GUÉRIN Claire (2012), La traduction littéraire en France, 1840-1915 : un projet d'histoire quantitative, transnationale et cartographique, Artl@S Bulletin, Vol. 1, no. 1 (2012), Article 5. En ligne : $<$ https://docs.lib.purdue.edu/artlas/vol1/iss1/5/> [consulté le 24 juillet 2018].

Wolf Nelly (1995), Une littérature sans histoire : essai sur le nouveau roman, Genève, Droz. 


\section{Annexe}

Tableau 1. Titres traduits et publiés (saisie chronologique)

\begin{tabular}{|c|c|c|c|c|c|c|c|c|c|c|}
\hline 1958-1962 & 1963-1967 & 1968-1972 & 1973-1977 & 1978-1982 & 1983-1987 & 1988-1992 & 1993-1997 & 1998-2002 & 2003-2007 & $2008-2016$ \\
\hline $\begin{array}{l}\text { Beckett } \\
\text { Nouvelles }\end{array}$ & $\begin{array}{l}\text { Pinget } \\
\text { Autour de } \\
\text { Mortin }\end{array}$ & $\begin{array}{l}\text { Cayrol } \\
\text { Je vivrai } \\
\text { l'amour des } \\
\text { autres }\end{array}$ & $\begin{array}{l}\text { Beckett } \\
\text { Premier } \\
\text { amour }\end{array}$ & $\begin{array}{l}\text { Beckett } \\
\text { Prose }\end{array}$ & $\begin{array}{l}\text { Beckett } \\
\text { Molloy (1) }\end{array}$ & $\begin{array}{l}\text { Duras Amant } \\
\text { (2) }\end{array}$ & $\begin{array}{l}\text { Beckett } \\
\text { Premier } \\
\text { amour } \\
\text { Molloy }\end{array}$ & $\begin{array}{l}\text { Duras } \\
\text { Moderato } \\
\text { cantabile }\end{array}$ & $\begin{array}{l}\text { Beckett } \\
\text { Molloy (2) }\end{array}$ & $\begin{array}{l}\text { Beckett } \\
\text { Compagnie } \\
\text { Soubresauts }\end{array}$ \\
\hline $\begin{array}{l}\text { Butor } \\
\text { Emploi du } \\
\text { temps }\end{array}$ & $\begin{array}{l}\text { Robbe- } \\
\text { Grillet } \\
\text { La maison } \\
\text { de rendez- } \\
\text { vous } \\
\end{array}$ & & $\begin{array}{l}\text { Cayrol } \\
\text { Corps } \\
\text { étrangers }\end{array}$ & $\begin{array}{l}\text { Simon } \\
\text { Route des } \\
\text { Flandres }\end{array}$ & $\begin{array}{l}\text { Robbe- } \\
\text { Grillet Djinn }\end{array}$ & $\begin{array}{l}\text { Simon } \\
\text { L'invitation }\end{array}$ & $\begin{array}{l}\text { Beckett } \\
\text { Molloy (1) }\end{array}$ & $\begin{array}{l}\text { Duras } \\
\text { Écrire }\end{array}$ & $\begin{array}{l}\text { Robbe- } \\
\text { Grillet } \\
\text { Gommes }\end{array}$ & $\begin{array}{l}\text { Sarraute } \\
\text { Tropismes }\end{array}$ \\
\hline $\begin{array}{l}\text { Robbe- } \\
\text { Grillet } \\
\text { Gommes }\end{array}$ & $\begin{array}{l}\text { Butor } \\
\text { Répertoire } \\
\text { (extraits) }\end{array}$ & & $\begin{array}{l}\text { Cayrol } \\
\text { Je l'entends } \\
\text { encore }\end{array}$ & & $\begin{array}{l}\text { Duras Amant } \\
\text { (1) }\end{array}$ & & $\begin{array}{l}\text { Duras } \\
\text { Amant (2) }\end{array}$ & & & \\
\hline $\begin{array}{l}\text { Sarraute } \\
\text { Portrait }\end{array}$ & & & $\begin{array}{l}\text { Robbe- } \\
\text { Grillet } \\
\text { Jalousie }\end{array}$ & & & & $\begin{array}{l}\text { Duras } \\
\text { L'amant de } \\
\text { la Chine du } \\
\text { Nord } \\
\end{array}$ & & & \\
\hline $\begin{array}{l}\text { Butor } \\
\text { Modification }\end{array}$ & & & $\begin{array}{l}\text { Sarraute } \\
\text { Vous les } \\
\text { entendez } \\
\end{array}$ & & & & $\begin{array}{l}\text { Robbe- } \\
\text { Grillet } \\
\text { Le voyeur }\end{array}$ & & & \\
\hline $\begin{array}{l}\text { Cayrol } \\
\text { Déménagem } \\
\text { ent }\end{array}$ & & & & & & & $\begin{array}{l}\text { Beckett } \\
\text { Malone } \\
\text { meurt }\end{array}$ & & & \\
\hline $\begin{array}{l}\text { Duras } \\
\text { Barrage }\end{array}$ & & & & & & & $\begin{array}{l}\text { Duras } \\
\text { La maladie } \\
\text { de la mort } \\
\end{array}$ & & & \\
\hline $\begin{array}{l}\text { Duras } \\
\text { Dix heures } \\
\text { et demie } \\
\end{array}$ & & & & & & & & & & \\
\hline
\end{tabular}

